Monatshefte für Mathematik 81, 15--40 (1976)

(C) by Springer-Verlag 1976

\title{
Zwei Klassen lokalkompakter maximal fastperiodischer Gruppen
}

\author{
Von \\ Detlev Poguntke, Bielefeld
}

(Eingegangen am 29. Juli 1974)

\begin{abstract}
Two Classes of Locally Compact Maximally Almost Periodic Groups. In this paper we study the class $\mathfrak{A}$ of all locally compact groups $G$ with the property that for each closed subgroup $H$ of $G$ there exists a pair of homomorphisms into a compact group with $H$ as coincidence set, and the class $\mathfrak{D}$ of all locally compact groups $G$ with the property that finite dimensional unitary representations of subgroups of $G$ can be extended to finite dimensional representations of $G$. It is shown that [MOORE]-groups (every irreducible unitary representation is finite dimensional) have these two properties. A solvable group in $\mathfrak{D}$ is a [MOORE]-group. Moreover, we prove a structure theorem for Lie groups in the class [MOORE], and show that compactly generated Lie groups in [MOORE] have faithful finite dimensional unitary representations.
\end{abstract}

\section{Bezeichnungen}

Es sei

[MAP] die Klasse der lokalkompakten maximal fastperiodischen Gruppen, d. h. derjenigen lokalkompakten Gruppen, die einen injektiven stetigen Homomorphismus in eine kompakte Gruppe zulassen,

[MOORE] die Klasse der lokalkompakten Gruppen, deren irreduzible unitäre Darstellungen sämtlich endlichdimensional sind,

[TAK] die Klasse derjenigen $G \in[\mathrm{MAP}]$, für die $\bar{G}^{\prime}$ kompakt ist (mit $\bar{G}^{\prime}$ wird stets der Abschluß der Kommutatorgruppe bezeichnet).

[Z] die Klasse der lokalkompakten Gruppen $G$, für die $G / Z G$ kompakt ist ( $Z G$ bezeichnet stets das Zentrum von $G$ ).

Für eine lokalkompakte Gruppe $G$ sei $r: G \rightarrow b G$ eine Bohrkompaktifizierung von $G$, d. h. $r$ ist ein dichter stetiger Homomorphis- 
mus von $G$ in die kompakte Gruppe $b G$, und zu jedem stetigen Homomorphismus $f$ von $G$ in eine kompakte Gruppe $K$ existiert genau ein stetiger Homomorphismus $f^{\prime}: b G \rightarrow K$ mit $f^{\prime} r=f . G$ ist genau dann eine [MAP]-Gruppe, wenn $r$ injektiv ist.

\section{§ 1. Einleitung}

Für kompakte Gruppen gelten die beiden folgenden Sätze:

(1.1) Satz. (vgl. etwa (1.1) in [13]). Ist $H$ eine abgeschlossene Untergruppe einer kompakten Gruppe $G$ und ist $p$ ein stetiger Homomorphismus von $H$ in die unitäre Gruppe $U(V)$ des endlichdimensionalen komplexen Hilbertraumes $V$ (ist $V$ ein komplexer Hilbertraum, so bezeichne $U(V)$ im folgenden stets dessen unitöre Gruppe), so gibt es einen $V$ umfassenden endlich-dimensionalen komplexen Hilbertraum $W$ (das innere Produkt auf $V$ ist nicht notwendig die Einschränkung des inneren Produktes auf $W)$ und einen stetigen Homomorphismus $\psi$ von $G$ in $U(W)$ mit $\psi(h)(v)=\varphi(h)(v)$ für $(h, v) \in H \times V, d . h . V$ ist ein H-Unterraum des G-Moduls $(W, \psi) . D a$ das orthogonale Komplement $V^{\perp}$ von $V$ in $W$ ebenfalls ein $H$-Unterraum von $(W, \psi)$ ist, gilt $\psi(h)=\varphi(h) \oplus \varphi^{\prime}(h)$ für $h \in H$, wobei $\varphi^{\prime}$ ein stetiger Homomorphismus von $H$ in die unitäre Gruppe von $V^{\perp} i s t$.

(1.2) Satz. (vgl. (1.4) bzw. Bemerkung dazu in [13]). Ist H eine abgeschlossene Untergruppe einer kompakten Gruppe $G$, so gibt es eine kompakte Gruppe $L$ und für $i=1,2$ stetige Homomorphismen $u_{i}: G \rightarrow L$ mit $H=\left\{x \in G \mid u_{1}(x)=u_{2}(x)\right\}$.

Ist $G$ eine [MAP]-Gruppe und $H$ eine kompakte Untergruppe von $G$, so folgt aus (1.1), daß man jede endlichdimensionale unitäre Darstellung von $H \mathrm{im}$ Sinne von (1.1) auf $G$,fortsetzen" kann (vgl. Theorem 5.1 in [6]). Mit Hilfe dieses Ergebnisses (und des Struktursatzes für [Z]-Gruppen) wird dann in [6] gezeigt, daß es zu jedem Charakter $\lambda$ des Zentrums $Z G$ einer [Z]-Gruppe $G$ eine stetige endlichdimensionale irreduzible unitäre Darstellung $\varrho$ von $G$ in $V$ gibt mit $\varrho(x)=\lambda(x) 1_{V}$ für $x \in Z G$ (in anderen Worten: (1.1) bleibt richtig, wenn $G$ eine [Z]-Gruppe und $H=Z G$ ist). Ferner bleiben (1.1) und (1.2) richtig, wenn man "kompakten Gruppe $G^{\prime \prime}$ jeweils durch „lokalkompakten abelschen Gruppe $G^{\text {“c }}$ ersetzt. Wir wollen nun in dieser Arbeit die beiden folgenden Klassen lokalkompakter Gruppen untersuchen.

(1.3) Definition. Es sei $\mathscr{O}$ die Klasse aller lokalkompakten Gruppen $G$ mit der Eigenschaft: Ist $H$ eine abgeschlossene Unter- 
gruppe von $G, V$ ein endlichdimensionaler komplexer Hilbertraum und $\varphi$ ein stetiger Homomorphismus von $H$ in $U(V)$, so gibt es einen $V$ umfassenden, endlichdimensionalen komplexen Hilbertraum $W$ und einen stetigen Homomorphismus $\psi: G \rightarrow U(W)$ mit $\psi(h)(v)=\varphi(h)(v)$ fürr $(h, v) \in H \times V$.

(1.4) Bemerkung. Da jede endlichdimensionale unitäre Darstellung direkte Summe irreduzibler Darstellungen ist, kann man in der Definition hinzufügen, daß $\varphi$ und $\psi$ irreduzibel sind, ohne dadurch an der Klasse $\mathfrak{P}$ etwas zu ändern.

(1.5) Definition. Es sei $\mathfrak{A}$ die Klasse aller lokalkompakten Gruppen $G$ mit der Eigenschaft: Ist $H$ eine abgeschlossene Untergruppe von $G$, so gibt es eine kompakte Gruppe $L$ und für $i=1,2$ stetige Homomorphismen $u_{i}: G \rightarrow L$ mit $H=\left\{x \in G \mid u_{1}(x)=u_{2}(x)\right\}$.

In $\S 2$ werden einige „Relativsätze" bewiesen. Es wird gezeigt, daß die Klassen $\mathfrak{A}$ und $\mathscr{P}$ abgeschlossen sind bezüglich der Bildung abgeschlossener Untergruppen, endlicher Erweiterungen und Quotienten (insbesondere liegt nicht jede diskrete [MAP]-Gruppe in $\mathfrak{A}$ bzw. $\mathfrak{D}$, denn jede freie diskrete Gruppe ist eine [MAP]-Gruppe, aber nicht jede diskrete Gruppe ist eine [MAP]-Gruppe, und die Gruppen in $\mathfrak{A}$ bzw. $\mathfrak{O}$ sind - wie wir bald sehen werden - [MAP]Gruppen). Ferner wird gezeigt: Ist $G$ eine [MAP]-Gruppe und $K$ ein kompakter Normalteiler in $G$ derart, daß $G / K$ in $\mathscr{D}$ bzw. in $\mathfrak{A} \cap \mathfrak{D}$ liegt, so liegt auch $G$ in $\mathscr{D}$ bzw. in $\mathfrak{A} \cap \mathscr{P}$ (in einer folgenden Arbeit werden wir sehen, daß der entsprechende Satz für die Klasse 2 falsch ist). Daraus folgt dann in $\S 3$ unmittelbar, daß jede [TAK]-Gruppe (und damit auch jede [Z]-Gruppe) in $\mathfrak{A} \cap \mathscr{D}$ liegt, womit das oben zitierte Resultat von Grosser und MoszowITZ über das Liften von Charakteren des Zentrums von [Z]Gruppen wesentlich verallgemeinert ist. Ferner wird in $\$ 3$ ein einfacher Beweis des Robertsonschen Satzes über die Struktur von [MOORE]-Gruppen gegeben, aus dem dann folgt, daß jede [MOORE]-Gruppe in $\mathfrak{A} \cap \mathfrak{D}$ liegt. Es wird ferner gezeigt, daß jede Liegruppe in [MOORE] einen offenen Normalteiler $H$ von endlichem Index besitzt, für den $H=\bar{H}^{\prime} Z H$ gilt. Danach wird die Struktur der auflösbaren Gruppen in $\mathscr{D}$ untersucht. Es zeigt sich, daß eine auflösbare lokalkompakte Gruppe genau dann in $\mathfrak{D}$ liegt, wenn sie eine [MOORE]-Gruppe ist. Um im folgenden kürzer formulieren zu können, vereinbaren wir: Sind $G_{1}$ und $G_{2}$ topologische Gruppen, so beinhalte die Aussage , $f$ ist ein Homomorphismus von $G_{1}$ in $G_{2}{ }^{\text {"c }}$ 
stets, daß $f$ auch stetig ist. Ferner wollen wir unter einer Darstellung einer topologischen Gruppe stets eine stetige unitäre Darstellung in einem komplexen Hilbertraum verstehen. Die Untersuchungen dieser Arbeit werden in einer Arbeit mit dem Titel „Chu-Dualität und zwei Klassen maximal fastperiodischer Gruppen“ fortgeführt.

\section{§ 2. Endliche Erweiterungen, Untergruppen und Quotienten von Gruppen in $\mathfrak{A}$ oder $\mathfrak{D}$}

Zunächst werden Kriterien dafür angegeben, daß eine Gruppe in $\mathfrak{A}$ bzw. in $\mathfrak{D}$ liegt. Es handelt sich dabei um einfache, aber im folgenden recht nützliche Umformulierungen der Definitionen der Klassen $\mathfrak{A}$ und $\mathfrak{D}$.

(2.1) Satz. Seien $G$ eine lokalkompalte Gruppe, $H$ eine abgeschlossene Untergruppe von $G$ und $r: G \rightarrow b G$ eine Bohrlompaltifizierung von $G$.

Dann sind äquivalent:

(1) Es gibt eine kompalte Gruppe $L$ und Homomorphismen $u_{i}: G \rightarrow L$ für $i=1,2$ mit $H=\left\{x \in G \mid u_{1}(x)=u_{2}(x)\right\}$.

(2) Es gitt $H=r^{-1}\left(r(H)^{-}\right)$.

(3) $Z u$ jedem $x \in G \backslash H$ gibt es einen endlichdimensionalen komplexen Hilbertraum $V$ und zwei Homomorphismen $\varphi$ und $\psi$ von $G$ in $U(V)$ mit $\varphi(x) \neq \psi(x)$, aber $\varphi(h)=\psi(h)$ für alle $h \in H$.

(4) $Z u$ jedem $x \in G \backslash H$ gibt es eine kompalcte Gruppe $L_{x}$ und Homomorphismen $\varphi_{x}$ und $\psi_{x}$ von $G$ in $L_{x}$ mit $\varphi_{x}(x) \neq \psi_{x}(x)$, aber $\varphi_{x}(h)=\psi_{x}(h)$ für alle $h \in H$.

Insbesondere liegt $G$ genau dann in $\mathfrak{A}$, wenn für jede abgeschlossene Untergruppe $H$ von $G$ eine der Bedingungen (1), (2), (3) oder (4) erfüllt ist.

(2.2) Bemerkung. Jede Gruppe in $\mathfrak{A}$ ist eine [MAP]-Gruppe: man wähle in (2) für $H$ die triviale Untergruppe.

Beweis des Satzes (1) $\Rightarrow$ (2): Wegen der universellen Eigenschaft von $r$ gibt es eindeutig Homomorphismen $\hat{u}_{i}: b G \rightarrow L$ für $i=1,2$ mit $\hat{u}_{i} r=u_{i}$. Die Inklusion $H \subset r^{-1}\left(r(H)^{-}\right)$gilt trivialerweise. Sei nun $x \in r^{-1}\left(r(H)^{-}\right)$. Dann liegt $r(x)$ in $r(H)^{-}$. Auf $r(H)^{-}$stimmen $\hat{u}_{1}$ und $\hat{u}_{2}$ aber aus Stetigkeitsgründen überein, also gilt $\hat{u}_{1} r(x)=\hat{u}_{2} r(x)$ oder $u_{1}(x)=u_{2}(x)$, woraus sich $x \in H$ ergibt.

(2) $\Rightarrow$ (3): Wegen (2) liegt $r(x)$ für $x \in G \backslash H$ nicht in $r(H)^{-}$. Nach (1.4) von [13] gibt es dann aber einen endlichdimensionalen 
komplexen Hilbertraum $V$ sowie zwei Homomorphismen $\hat{\varphi}$ und $\hat{\psi}$ von $b G$ in $U(V)$, die auf $r(H)^{-}$übereinstimmen, an der Stelle $r(x)$ aber verschiedene Werte annehmen. Die Homomorphismen $\varphi=\hat{\varphi} r$ und $\psi=\hat{\psi} r$ leisten dann das Gewünschte.

$(3) \Rightarrow(4)$ : trivial.

$(4) \Rightarrow(1)$ : Für jedes $x \in G \backslash H$ seien $L_{x}, \varphi_{x}$ und $\psi_{x}$ mit den in (3) angegebenen Eigenschaften gewählt. Dann ist $L:=\prod_{x \in G \backslash B} L_{x}$ eine kompakte Gruppe. Die Homomorphismen $\varphi_{x}$ bzw. $\psi_{x}, x \in G \backslash H$, definieren Homomorphismen $u_{1}$ bzw. $u_{2}$ von $G$ in dieses Produkt. Offensichtlich haben $u_{1}$ und $u_{2}$ die in (1) geforderten Eigenschaften.

(2.3) Satz. Seien $G$ eine lokalkompakte Gruppe, $H$ eine abgeschlossene Untergruppe von $G$ und $r: G \rightarrow b G$ eine Bohrkompaktifizierung von $G$. Dann sind äquivalent:

(1) Die Einschränkung (und Coeinschränkung) $r_{H}: H \rightarrow r(H)^{-}$ von $r$ ist eine Bohrkompaktifizierung von $H$.

(2) Bezeichnen $s: H \rightarrow b H$ eine Bohrkompaktifizierung von $H$ und $u: H \rightarrow G$ den Inklusionshomomorphismus, so ist der eindeutig existierende Homomorphismus bu, der das Diagramm

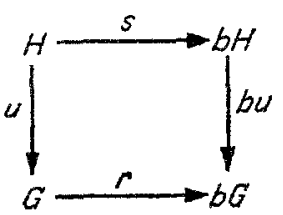

kommutativ ergänzt, injektiv.

(3) Ist $\varphi$ eine endlichdimensionale Darstellung von $H$ in $V$, so gibt es einen $V$ umfassenden, endlichdimensionalen komplexen Hilbertraum $W$ und eine Darstellung $\psi$ von $G$ in $W$ mit $\psi(h)(v)=\varphi(h)(v)$ für $(h, v) \in H \times V$.

(4) Ist $\varphi$ eine endlichdimensionale irreduzible Darstellung von $H$ in $V$, so gibt es einen $V$ umfassenden, endlichdimensionalen komplexen Hilbertraum $W$ und eine irreduzible Darstellung $\psi$ von $G$ in $W$ mit $\psi(h)(v)=\varphi(h)(v)$ fiir $(h, v) \in H \times V$.

Insbesondere liegt $G$ genau dann in $\mathscr{D}$, wenn für jede abgeschlossene Untergruppe $H$ von $G$ eine der Bedingungen (1), (2), (3) oder (4) erfuillt ist.

(2.4) Bemerkung. Jede Gruppe in $\mathscr{D}$ ist eine [MAP]-Gruppe: zu jedem Element $x$ in $G$ wähle man eine $x$ umfassende abgeschlossene abelsche Untergruppe $H$ und verwende (1). 
Beweis des Satzes (2) $\Rightarrow(1)$ : Unter Verwendung der Tatsache, daß $s$ eine dichte stetige Abbildung ist, weist man ohne Mühe nach, daß $b u(b H)=r(H)^{-}$ist (dies gilt auch, wenn $b u$ nicht injektiv ist). Dann induziert $b u$ wegen (2) durch Coeinschränkung einen Isomorphismus $f$ von $b H$ auf $r(H)^{-}$mit $f s=r_{H}$, folglich ist $r_{H}$ eine Bohrkompaktifizierung von $H$.

$(1) \Rightarrow(3): V$ und $\varphi$ mögen die in (3) angegebenen Eigenschaften besitzen. Wegen (1) gibt es einen Homomorphismus $\hat{\varphi}: r(H)^{-} \rightarrow U(V)$ mit $\hat{\varphi} r_{H}=\varphi$. Zu $\hat{\varphi}$ gibt es aber nach (1.1) in [13] einen $V$ umfassenden, endlichdimensionalen komplexen Hilbertraum $W$ und einen Homomorphismus $\hat{\psi}$ von $b G$ in $U(W)$ mit $\hat{\psi}(h)(v)$ für $(h, v) \in r(H)^{-} \times V$. Offenbar hat dann $\psi:=\hat{\psi} r$ die in (3) geforderte Eigenschaft.

$(3) \Rightarrow(2)$ : Nehmen wir an, es gebe ein vom Einselement verschiedenes Element $a$ im Kern von $b u$. $\mathrm{Zu}$ diesem $a$ existieren nach dem Theorem und Peter und Weyl ein endlichdimensionaler komplexer Hilbertraum $V$ und ein Homomorphismus $\hat{\varphi}: b H \rightarrow U(V)$ mit $\hat{\varphi}(a) \neq 1_{V}$; dann sei $\varphi:=\hat{\varphi} s$. Zu $\varphi$ existieren wegen (3) ein $V$ umfassender, endlich-dimensionaler komplexer Hilbertraum $W$ und eine Darstellung $\psi$ von $G$ in $W$ mit $\psi(h)(v)=\varphi(h)(v)$ für $(h, v) \in H \times V$. Wegen der universellen Eigenschaft von $r$ gibt es einen Homomorphismus $\hat{\psi}: b G \rightarrow U(W)$ mit $\hat{\psi} r=\psi$. Aus Stetigkeitsgründen ist $\hat{\psi}(y)(V)=V$ für alle $y \in r(H)^{-}$. Bezeichnet dann Aut $(V)$ die Gruppe aller Vektorraum-Isomorphismen von $V$ auf sich, so ist wegen. $b u(b H)=r(H)^{-}$durch $(\tilde{\varphi}(x))(v)=(\hat{\varphi}(b u(x)))(v)$ für $(x, v) \in b H \times V$ ein Homomorphismus $\tilde{\varphi}$ von $b H$ in Aut $(V)$ definiert. Wir zeigen nun, $\mathrm{da} ß \tilde{\varphi}$ und $\hat{\varphi}$ auf $s(H)$ übereinstimmen (wobei $\hat{\varphi}$ als Homomorphismus von $b H$ in $\operatorname{Aut}(V)$ aufzufassen ist). Für $h \in H$ und $v \in V$ ist nämlich

$$
\begin{gathered}
\tilde{\varphi}(s(h))(v)=\hat{\psi}((b u s)(h))(v)=\hat{\psi}(r(h))(v)=\psi(h)(v)= \\
=\varphi(h)(v)=\hat{\varphi}(s(h))(v) .
\end{gathered}
$$

Wegen der Stetigkeit von $\tilde{\varphi}$ und $\hat{\varphi}$ ist dann $\tilde{\varphi}=\hat{\varphi}$, da $s(H)$ eine dichte Teilmenge von $b H$ ist. Insbesondere ist $\tilde{\varphi}(a)=\hat{\varphi}(a)$, damit haben wir einen Widerspruch erhalten, denn es ist $\hat{\varphi}(a) \neq 1_{V}$, aber $\tilde{\varphi}(a)=1_{V}$. Die Aussagen (3) und (4) sind äquivalent, weil jede unitäre endlich-dimensionale Darstellung direkte Summe irreduzibler Darstellungen ist.

Man kann ohne Schwierigkeiten zeigen, daß die Klassen $\mathfrak{A}$ und (D) abgesehlossen sind bezüglich der Bildung abgeschlossener Untergruppen. 
(2.5) Satz. Sei U eine abgeschlossene Untergruppe einer lokalkompakten Gruppe $G$. Ist dann $G$ in $\mathfrak{A}$ bzw. in $\mathfrak{D}$, so liegt auch $U$ in $\mathfrak{A} b z w$. in $\mathfrak{D}$.

Beweis. Ist nämlich $G$ in $\mathfrak{A}$ und ist $H$ eine abgeschlossene Untergruppe von $U$, so ist $H$ eine abgeschlossene Untergruppe von $G$. Daher gibt es eine kompakte Gruppe $L$ und Homomorphismen $u_{1}, u_{2}: G \rightarrow L$, die genau auf $H$ übereinstimmen. Die Einschränkungen von $u_{1}$ und $u_{2}$ auf $U$ sind dann zwei Homomorphismen von $U$ in $L$, die genau auf $H$ übereinstimmen. Genauso einfach ist der Beweis dafür, daß $U$ in $\mathscr{D}$ liegt, falls $G$ diese Eigenschaft besitzt.

Die folgenden Sätze sind ein wenig schwieriger zu beweisen. Wir schicken diesen einige Lemmata voraus.

(2.6) Lemma. Seien $G$ eine topologische Gruppe und $N$ ein abgeschlossener Normalteiler in $G$. Ist dann $r: G \rightarrow b G$ eine Bohrkompaktifizierung von $G$, so ist $r(N)^{-}$normal in $b G$. Es gibt genau einen Homomorphismus $\hat{r}$ derart, daß das Diagramm.

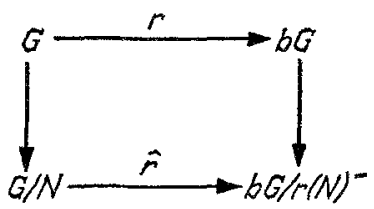

kommutiert, wobei die senkrechten Pfeile die natürlichen Homomorphismen symbolisieren. Ferner ist $\hat{r}$ eine Bohrkompaktifizierung von $G / N$.

Beweis. Da $r$ eine dichte Abbildung ist, ist $r(N)^{-}$ein Normalteiler in $b G$. Die Existenz von $\hat{r}$ ist evident. Die universelle Eigenschaft von $\hat{r}$ ergibt sich unmittelbar aus der universellen Eigenschaft von $r$.

(2.7) Lemma. Sei $G$ eine topologische Gruppe und $N$ ein abgeschlossener Normalteiler in $G . K$ sei eine kompakte Gruppe, und $f: G \rightarrow K$ sei ein dichter Homomorphismus. Dann ist $f(N)^{-}$normal in $K$, und es gibt genau einen Homomorphismus $\hat{f}: G / N \rightarrow K / f(N)^{-}$ derart, daß das Diagramm

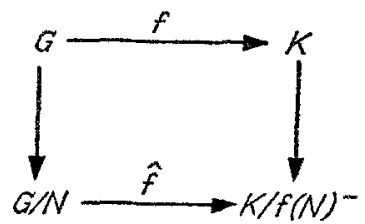


kommutiert. Sind f und die Einschränkung $f_{N}: N \rightarrow f(N)^{-}$von $f$ Bohrkompaktifizierungen von $N$ bzw. $G / N$, so ist $f$ eine Bohrkompaktifizierung von $G$.

Bevor wir (2.7) beweisen, wollen wir eine in der Folge verwendete spezielle Version davon in (2.8) formulieren.

(2.8) Lemma. Seien $G$ eine topologische Gruppe, $U$ eine abgeschlossene Untergruppe von $G$ und $N$ ein abgeschlossener Normalteiler von $U$. Ferner sei $r: G \rightarrow b G$ eine Bohrkompaltifizierung von $G$; der eingeschränlte Homomorphismus $r_{N}: N \rightarrow r(N)$ - und der induzierte Homomorphismus $U / N \rightarrow r(U)-\mid r(N)^{-}$seien Bohrkompaktifizierungen von $N$ bzw. U/N. Dann ist $r_{U}: U \rightarrow r(U)^{-}$eine Bohrkompaktifizierung von $U$.

Beweis von (2.7). Die Normalteilereigenschaft von $f(N)^{-}$und die Existenz von $\hat{f}$ sind klar. Der wesentliche und auch nicht ganz so einfache Teil ist, daß $f$ eine Bohrkompaktifizierung von $G$ ist.

Sei dazu $r: G \rightarrow b G$ eine Bohrkompaktifizierung von $G$. Wegen der universellen Eigenschaft von $r$ gibt es $w: b G \rightarrow K$ mit $w r=f$. Da $f$ dicht ist, ist es auch $w$. Mithin ist $w$ surjektiv. Es bleibt zu zeigen, daß $w$ auch injektiv ist. Wie man leicht nachweist, gilt $f(N)^{-}=$ $=w\left(r(N)^{-}\right)$. Daher gibt es $\hat{w}$ derart, daß

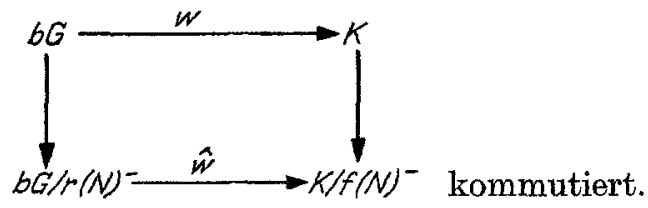

Bezeichnet man mit $\hat{r}: G / N \rightarrow b G / r(N)^{-}$den Homomorphismus aus (2.6), so kommutiert auch

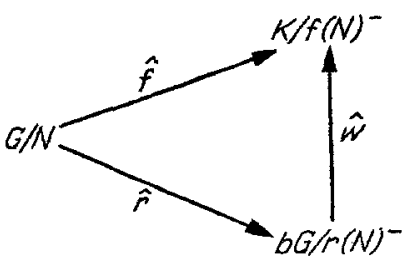

Da nach Voraussetzung $\hat{f}$ eine Bohrkompaktifizierung von $G / N$ ist, gibt es $v: K / f(N)^{-} \rightarrow b G / r(N)^{-}$mit $v \hat{f}=\hat{r}$. Man weist dann sofort nach, daß $w$ und $v$ invers zueinander sind. Daraus folgt unmittelbar, daß Kern $w$ in $r(N)$ - liegt.

Aus $f(N)^{-}=w\left(r(N)^{-}\right)$ergibt sich, daB man eine Einschränkung $w_{N}: r(N)^{-} \rightarrow f(N)^{-}$von $w$ definieren kann. Wie gehabt, seien 
$r_{N}: N \rightarrow r(N)^{-}$und $f_{N}: N \rightarrow f(N)^{-}$die Einschränkungen von $r$ und $f$. Dann kommutiert

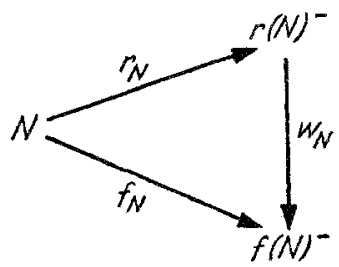

Da $f_{N}$ eine Bohrkompaktifizierung von $N$ ist, gibt es $u: f(N)^{-} \rightarrow$ $\rightarrow r(N)^{-}$mit $u f_{N}=r_{N}$. Aus $u w_{N} r_{N}=u f_{N}=r_{N}=1_{r(N)} r_{N}$ folgt $u w_{N}=1_{r(N)^{-}}$, da $r_{N}$ dicht ist. Zusammen mit Kern $w \subset r(N)^{-}$ergibt sich nun, daß Kern $w$ trivial ist.

(2.9) Lemma. Sei G eine topologische Gruppe mit Bohrkompaktifizierung $r: G \rightarrow b G$. Ist dann $N$ ein abgeschlossener Normalteiler in $G$ von endlichem Index, so gilt $r^{-1}\left(r(N)^{-}\right)=N$, und die Einschränkung $r_{N}: N \rightarrow r(N)^{-}$von $r$ ist eine Bohrkompaktifizierung von $N$.

Beweis. Zunächst beweisen wir, daß $r_{N}$ eine Bohrkompaktifizierung von $N$ ist. Nach bekannten Sätzen (im wesentlichen der Satz von Peter und Weyl, aus dem man sofort erhält, daß man jede kompakte Gruppe in ein Produkt unitärer Gruppen einbetten kann) genügt es dazu zu zeigen, daß zu einem Homomorphismus $\varphi$ von $N$ in die unitäre Gruppe $U(n)$ ein Homomorphismus $\hat{\varphi}$ mit $\hat{\varphi} r_{N}=\varphi$ existiert. Ist $s$ der Index von $N$ in $G$, so gibt es zu $\varphi$ einen Homomorphismus $\varphi^{*}$ von $G$ in $U(n s)$ derart, daß für $x \in N$ die Matrix $\varphi^{*}(x)$ von der Form

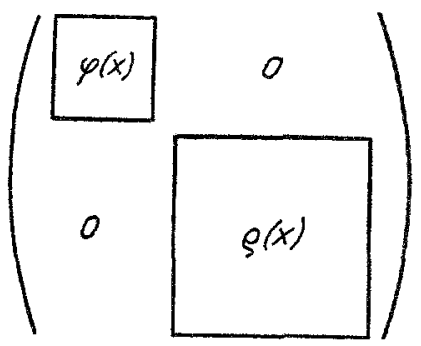

ist, wobei $\varrho$ ein Homomorphismus von $N$ in $U(n(s-1))$ ist (das ist nichts anderes als die aus der Theorie der Darstellungen endlicher Gruppen bekannte Konstruktion der induzierten Darstellung, vgl. etwa [2], p. 79). 
Zu $\varphi^{*}$ existiert nun ein Homomorphismus $\hat{\psi}$ von $b G$ in $U(n s)$ mit $\hat{p} r=\varphi^{*}$. Aus Stetigkeitsgründen ist für $x \in r(N)^{-}$die Matrix $\hat{\psi}(x)$ von der Form

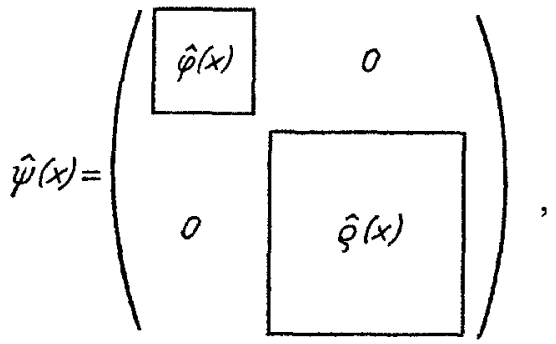

wobei $\hat{\varphi}$ und $\hat{\varrho}$ Homomorphismen von $r(N)^{-}$in $U(n)$ bzw. $U(n(s-1))$ sind. Offenbar ist dann $\hat{\varphi} r_{N}=\varphi$.

Nun weisen wir die Gleichung $N=r^{-1}\left(r(N)^{-}\right)$nach. Auf Grund von (2.6) ist der induzierte Homomorphismus $\hat{r}$ in

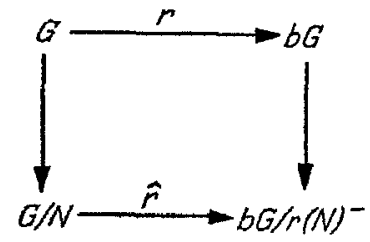

eine Bohrkompaktifizierung von $G / N$. Da $G / N$ endlich ist, ist $\hat{r}$ ein Isomorphismus, und mithin gilt die behauptete Gleichheit.

Mit Hilfe von (2.6) und den in (2.1) und (2.3) angegebenen Kriterien kann man zeigen, daß die Klassen $\mathfrak{A}$ und $\mathfrak{D}$ abgeschlossen bezüglich der Bildung von Quotienten sind.

(2.10) Satz. Ist $G$ in $\mathfrak{A}$ und ist $N$ ein abgeschlossener Normalteiler in $G$, so liegt auch $G / N$ in $\mathfrak{A}$.

Beweis. Sei $r: G \rightarrow b G$ eine Bohrkompaktifizierung von $G$; $p: G \rightarrow G / N$ und $q: b G \rightarrow b G / r(N)^{-}$seien die natürlichen Homomorphismen. Der induzierte Homomorphismus $\hat{r}$

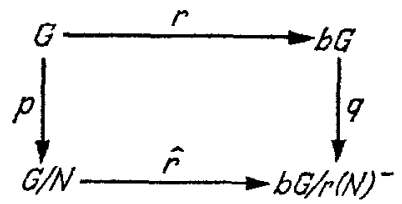

ist dann nach (2.6) eine Bohrkompaktifizierung von $G / N$. Wegen Kriterium (2) aus (2.1) genügt es zu zeigen, daß für eine abgeschlossene Untergruppe $H$ von $G / N$ die Gleichung $H=\hat{r}^{-1}\left(\hat{r}(H)^{-}\right)$gilt. 
Da $p^{-1}(H)$ in $G$ abgeschlossen ist, gilt nach Voraussetzung $p^{-1}(H)=r^{-1}\left(r\left(p^{-1}(H)\right)^{-}\right)$. Die Inklusion $H \subset \hat{r}^{-1}\left(\hat{r}(H)^{-}\right)$gilt stets.

Sei nun $x \in \hat{r}^{-1}\left(\hat{r}(H)^{-}\right)$. Dann gibt es $y \in G$ mit $p(y)=x$, und es gilt $\quad q r(y)=\hat{r} p(y)=\hat{r}(x) \in \hat{r}(H)^{-}=q\left(r\left(p^{-1}(H)\right)^{-}\right)$. Daraus folgt $r(y) \in r\left(p^{-1}(H)\right)^{-} \operatorname{Kern} q=r\left(p^{-1}(H)\right)^{-} \cdot r(N)^{-}=r\left(p^{-1}(H)\right)^{-}, \quad$ also $y \in r^{-1}\left(r\left(p^{-1}(H)^{-}\right)\right.$und folglich $y \in p^{-1}(H)$ bzw. $p(y)=x \in H$.

(2.11) Satz. Ist $G$ in $\mathscr{D}$ und $N$ ein abgeschlossener Normalteiler in $G$, so liegt auch $G / N$ in $\mathfrak{D}$.

Beweis. $r, \hat{r}, p, q$ mögen dieselbe Bedeutung wie im Beweis des vorigen Satzes haben. Sei $H$ eine abgeschlossene Untergruppe von $G / N$. Wegen Kriterium (1) aus (2.3) genügt es zu zeigen, daß die Einschränkung $\hat{r}_{H}: H \rightarrow \hat{r}(H)^{-}$eine Bohrkompaktifizierung von $H$ ist. Mit $p_{H I}: p^{-1}(H) \rightarrow H, q_{H}: r\left(p^{-1}(H)\right)^{-} \rightarrow q\left(r\left(p^{-1}(H)\right)^{-}\right)=\hat{r}(H)^{-}$ und $r_{H}: p^{-1}(H) \rightarrow r\left(p^{-1}(H)\right)^{-}$seien die Einschränkungen von $p, q$ bzw. $r$ bezeichnet; $r_{H}$ ist nach Voraussetzung eine Bohrkompaktifizierung von $p^{-1}(H)$. Sei nun $\varphi$ ein Homomorphismus von $H$ in die kompakte Gruppe $K$. Wir habenzu zeigen, daß es eindeutig (Eindeutigkeit ist natürlich trivial, da $\hat{r}_{H}$ dicht ist) $\tilde{\varphi}$ mit $\tilde{\varphi} \hat{r}_{H}=\varphi$ gibt.

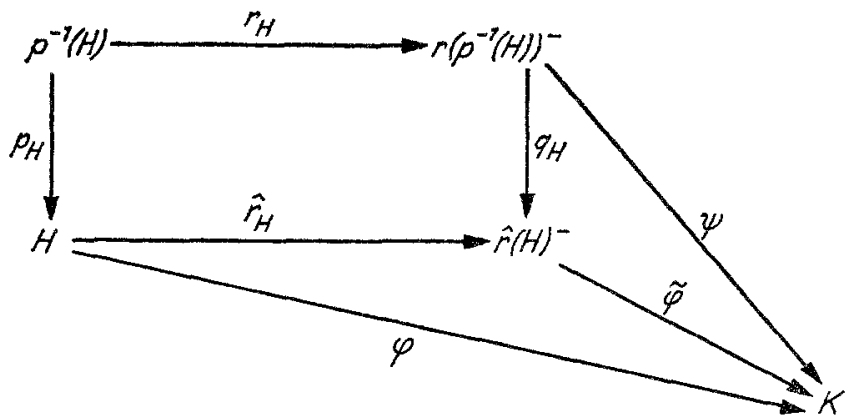

Da $r_{H}$ eine Bohrkompaktifizierung von $p^{-1}(H)$ ist, gibt es $\psi$ mit $\psi r_{H}=\varphi p_{H}$. Man überlegt sich dann leicht, daß $\psi$ über $q_{H}$ faktorisiert (d. h. daß es $\tilde{\varphi}$ mit $\tilde{\varphi} q_{H}=\psi$ gibt) und daß $\tilde{\varphi}$ die geforderte Eigenschaft besitzt.

Im nächsten Satz wird bewiesen, daß die Klassen $\mathfrak{A}$ und $\mathfrak{D}$ abgeschlossen gegen endliche Erweiterungen sind.

(2.12) Satz. $G$ sei eine lokalkompakte Gruppe, $N$ sei eine abgeschlossene Untergruppe in $G$ von endlichem Index. Dann liegt $G$ genau dann in $\mathfrak{A}(\mathfrak{D )})$, wenn $N$ in $\mathfrak{A}(\mathfrak{D})$ liegt.

Beweis. Die eine Richtung ist jeweils trivial, denn liegt $G$ in $\mathfrak{A}(\mathfrak{D})$, so liegt auch $N$ als abgeschlossene Untergruppe in $\mathfrak{A}(\mathfrak{D})$ 
nach (2.5). $G$ operiert auf dem Raum der Linksnebenklassen $G / N$, man hat also einen Homomorphismus von $G$ in die Gruppe der bijektiven Abbildungen von $G / N$ auf sich. Der Kern dieses Homomorphismus ist gerade gleich $M=\bigcap_{g \in G} g N g^{-1}$ und von endlichem Index in $G$. Ist dann $N$ in $\mathfrak{A}(\mathfrak{D})$, so liegt auch $M$ in $\mathfrak{A}(\mathfrak{D})$. Wir können also O. B. d. A. annehmen, daß $N$ normal in $G$ ist. Seien ferner $r: G \rightarrow b G$ eine Bohrkompaktifizierung von $G$ und $H$ eine abgeschlossene Untergruppe von $G$. Auf Grund von (2.1) und (2.3) genügt es zu zeigen:

(1) Ist $N$ in $\mathfrak{U}$, so gilt $r^{-1}\left(r(H)^{-}\right)=H$

(2) Ist $N$ in $\mathfrak{D}$, so ist die Einschränkung $r_{H}: H \rightarrow r(H)^{-}$von $r$ eine Bohrkompaktifizierung von $H$.

$\mathrm{Zu}$ (1): Aus (2.9) und der Voraussetzung über $N$ folgt leicht $r^{-1}\left(r(H \cap N)^{-}\right)=r_{N}^{-1}\left(r_{N}(H \cap N)^{-}\right)=H \cap N$.

Nun ist $H \cap N$ von endlichem Index in $H$, es gibt folglich $h_{1}, \ldots, h_{s} \in H$ mit $H=\bigcup_{i=1}^{s} h_{i}(H \cap N)$. Dann ist

$$
\begin{aligned}
r^{-1}\left(r(H)^{-}\right) & =r^{-1}\left(\left(\bigcup_{i=1}^{s} r\left(h_{i}\right) r(H \cap N)\right)^{-}\right)=r^{-1}\left(\bigcup_{i=1}^{s} r\left(h_{i}\right) r(H \cap N)^{-}\right)= \\
& =\bigcup_{i=1}^{s} h_{i}\left(r^{-1}\left(r(H \cap N)^{-}\right)\right)=\bigcup_{i=1}^{s} h_{i}(H \cap N)=H .
\end{aligned}
$$

$\mathrm{Zu}$ (2): Aus (2.9) und der Voraussetzung über $N$ folgt, daß die Einschränkung $H \cap N \rightarrow r(H \cap N)^{-}$eine Bohrkompaktifizierung von $H \cap N$ ist. Nach (2.8) genügt es dann aber zu zeigen, daß der induzierte Homomorphismus $s$ in

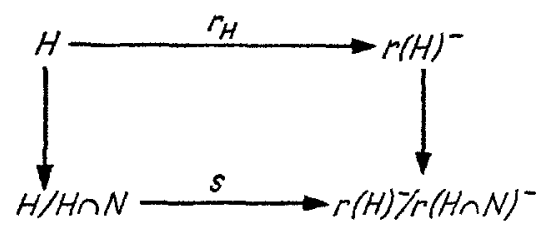

eine Bohrkompaktifizierung von $H / H \cap N$ ist.

Dazu betrachten wir den induzierten Homomorphismus $\hat{\gamma}$ in dem Diagramm

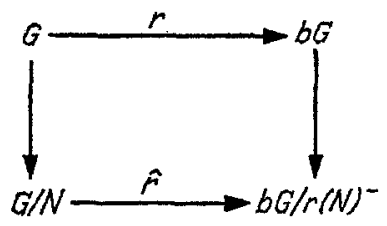


Da $G / N$ endlich und $\hat{r}$ nach (2.6) eine Bohrkompalktifizierung von $G / N$ ist, ist $\hat{r}$ sogar ein Isomorphismus und stellt einen Isomorphismus $t$ von $H N / N$ auf $\hat{r}(H N / N)=r(H N)-\operatorname{rr}(N)^{-}$her. Dann ist offenbar das Diagramm

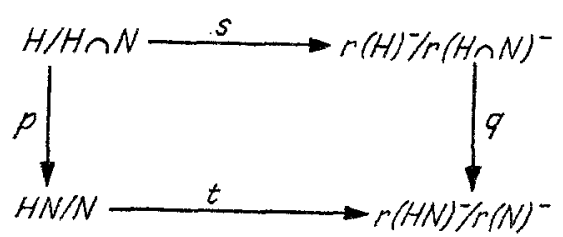

kommutativ, wobei $p$ und $q$ von den Einbettungen $H \rightarrow H N$ bzw. $r(H)^{-} \rightarrow r(H N)^{-}$herrühren.

Nun ist $s$ surjektiv, weil $s$ dicht und $H / H \cap N$ endlich ist. Ferner sind $p$ und $t$ Isomorphismen, insbesondere injektiv; dann muß aber auch $s$ injektiv sein. Folglich ist $s$ eine Bohrkompaktifizierung der endlichen Gruppe $H / H \cap N$, was zu zeigen war.

In den nächsten beiden Sätzen wollen wir hinreichende Kriterien dafür angeben, daß eine Gruppe in $\mathfrak{D}$ bzw. in $\mathfrak{A} \cap \mathfrak{D}$ liegt. Insbesondere ergibt sich dann aus diesen Kriterien, daß jede [TAK]-Gruppe in $\mathfrak{A} \cap \mathfrak{D}$ liegt.

(2.13) Satz. Sei $G$ eine [MAP]-Gruppe. $K$ sei ein kompakter Normalteiler in $G$, und $G / K$ liege in $\mathfrak{D}$. Dann liegt auch $G$ in $\mathfrak{D}$.

Beweis. Wir verwenden Kriterium (1) von (2.3). Seien $r: G \rightarrow b G$ eine Bohrkompaktifizierung von $G$ und $H$ eine abgeschlossene Untergruppe von $G$. Wir haben zu zeigen, daß die Einschränkung $r_{H}: H \rightarrow r(H)^{-}$eine Bohrkompaktifizierung von $H$ ist.

Setze $N:=H \cap K . N$ ist dann ein kompakter Normalteiler in $H$; insbesondere gilt $r(N)^{-}=r(N)$, und die eingeschränkte Abbildung $r_{N}: N \rightarrow r(N)$ jst ein Isomorphismus ( $G$ ist eine [MAP]-Gruppe, $r$ also injektiv); daher ist $r_{N}$ eine Bohrkompaktifizierung von $N$. Nach (2.8) sind wir fertig, wenn wir bewiesen haben, daß der induzierte Homomorphismus

$$
t: H / N \rightarrow r(H)-r(N)
$$

eine Bohrkompaktifizierung von $H / N$ ist. 
Seien mit $p: G \rightarrow G / K$ und $q: b G \rightarrow b G / r(K)$ die natürlichen Homomorphismen und mit $\hat{r}: G / K \rightarrow b G / r(K)$ der induzierte Homomorphismus bezeichnet. Dann ist das Diagramm

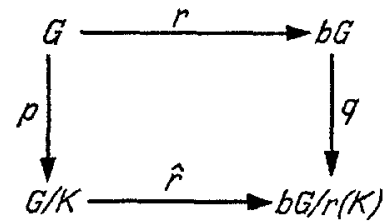

kommutativ, und $\hat{r}$ ist eine Bohrkompaktifizierung von $G / K$ (vgl. (2.6)). Da $K$ kompakt ist, ist $p$ eine abgeschlossene Abbildung. Insbesondere ist $p(H)$ abgeschlossen in $G / K$, und nach Voraussetzung über $G / K$ ist dann die Einschränkung $s: p(H) \rightarrow \hat{r}(p(H))^{-}$ von $\hat{r}$ eine Bohrkompaktifizierung von $p(H)$. Ferner ist das Diagramm

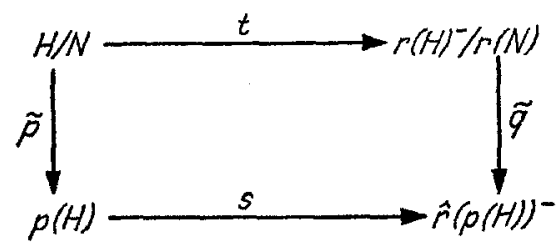

kommutativ, wobei $\tilde{p}$ und $\tilde{q}$ durch Einschränkung von $p$ bzw. $q$ und Ausfaktorisieren von $N$ bzw. $r(N)$ entstehen. Offenbar ist $\tilde{p}$ stetig und ein Isomorphismus der zugrundeliegenden diskreten Gruppen. Da nun $p: G \rightarrow G / K$ abgeschlossen ist, ist auch die Einschränkung $H \rightarrow p(H)$ und letztlich $\tilde{p}: H / N \rightarrow p(H)$ abgeschlossen; mithin ist $\tilde{p}$ offen and daher ein Isomorphismus topologischer Gruppen. Aus der Tatsache, daß $s: p(H) \rightarrow \hat{r}(p(H))^{-}$eine Bohrkompaktifizierung von $p(H)$ ist, folgt damit, daß $s \tilde{p}: H / N \rightarrow$ $\rightarrow \hat{r}(p(H))^{-}$eine Bohrkompaktifizierung von $H / N$ ist. Wenn man dann noch verwendet, daß $t$ eine dichte Abbildung ist, ergibt sich unmittelbar, da $ß \tilde{q}$ ein Isomorphismus und daß $t$ eine Bohrkompaktifizierung von $H / N$ ist, was zu beweisen war.

(2.14) Satz. Sei $G$ eine [MAP]-Gruppe. $K$ sei ein kompakter Normalteiler in $G$, und $G / K$ liege in $\mathfrak{A} \cap \mathfrak{D}$. Dann liegt auch $G$ in $\mathfrak{A} \cap \mathfrak{P}$.

Beweis. Auf Grund des vorigen Satzes genügt es zu zeigen, daß $G$ in $\mathfrak{A}$ liegt. Wir verwenden wiederum Kriterium (2) aus (2.1). 
Seien also $r: G \rightarrow b G$ eine Bohrkompaktifizierung von $G, H$ eine abgeschlossene Untergruppe von $G$ und $\hat{H}:=r^{-1}\left(r(H)^{-}\right)$. Wir haben die Inklusion $\hat{H} \subset H$ nachzuweisen. Mit $s: \hat{H} \rightarrow r(\hat{H})^{-}=r(H)^{-}$ sei die Einschränkung von $r$ bezeichnet. Da $G$ in $\mathfrak{D}$ liegt, ist $s$ eine Bohrkompaktifizierung von $\hat{H}$. Ferner sei $\widehat{K}:=K \cap \hat{H} ; \hat{K}$ ist ein kompakter Normalteiler in $\hat{H}$. Wie im Beweis des vorigen Satzes $(G \rightarrow G / K$ ist wieder eine abgeschlossene Abbildung) vergewissert man sich, daß $\hat{H} / \widehat{K}$ topologisch isomorph zu einer abgeschlossenen Untergruppe von $G / K$ ist, also liegt $\hat{H} / \hat{K}$ in $\mathfrak{A} \cap \mathfrak{D}$. Es gibt $\hat{s}$ derart, daß das Diagramm

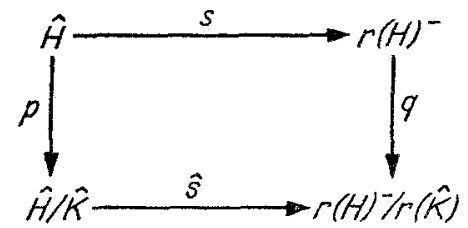

kommutiert, wobei $p$ und $q$ die natürlichen Homomorphismen bezeichnen. $p(H)$ ist eine abgeschlossene Untergruppe von $\hat{H} / \hat{K}$. Da $\hat{H} \mid \hat{K}$ in $\mathfrak{A} \cap \mathfrak{O}$ liegt und $\hat{s}$ eine Bohrkompaktifizierung von $\hat{H} / \hat{K}$ ist, gilt $\hat{s}^{-1}\left((\hat{s} p(H))^{-}\right)=p(H)$.

Nun ist aber $\hat{s}^{-1}\left((\hat{s} p(H))^{-}\right)=\hat{H} \mid \hat{K}$, und man erhält $\hat{H}=H \hat{K}$. Da $H \cap \widehat{K}$ normal in $H$ ist, ist $r(H \cap \hat{K})$ normal in $r(H), r(H \cap \widehat{K})^{-}=$ $=r(H \cap \hat{K})$ normal in $r(H)^{-}=r(\hat{H})^{-}$und mithin $H \cap \hat{K}$ normal in $\hat{H}$ ( $r$ ist injektiv!). Mit $a: \hat{H} \rightarrow \hat{H} / \hat{K} \cap H$ und $b: H \rightarrow H / H \cap \hat{K}$ seien die natürlichen Homomorphismen bezeichnet. Die Einbettung $j$ von $H$ in $\hat{H}$ induziert einen Homomorphismus $i$ derart, daß das Diagramm

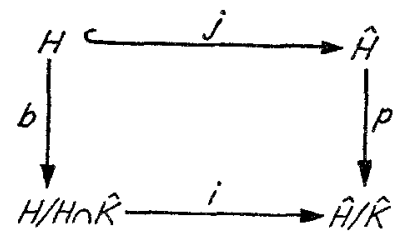

kommutiert.

Wegen $\hat{H}=H \hat{K}$ ist $i$ ein Isomorphismus diskreter Gruppen, wegen der Kompaktheit von $\hat{K}$ ist $i$ auch abgesehlossen und daher ein Isomorphismus topologischer Gruppen. Da $\hat{H}$ in $\mathscr{D}$ liegt, ist auch $\hat{H} / H \cap \hat{K}$ in $\mathscr{D}$, insbesondere ist $\hat{H} / H \cap \hat{K}$ eine [MAP]-Gruppe. 
Wähle nun einen injektiven Homomorphismus $\varphi$ von $\hat{H} / H \cap \widehat{K}$ in die kompakte Gruppe $L$. Dann sei $\psi:=\varphi a j, \psi$ faktorisiert über $b$, das heißt es gibt $\hat{\psi}$ mit $\hat{\psi} b=\psi$.

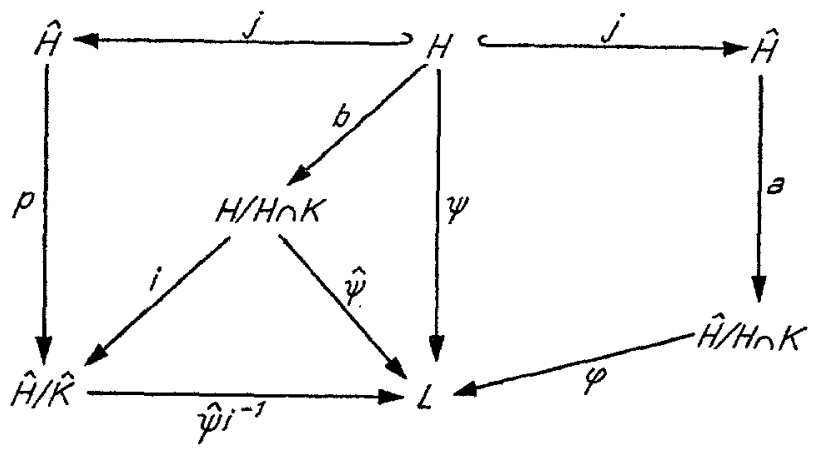

Offensichtlich gilt dann $\psi=\varphi a j=\hat{\psi} i^{-1} p j$.

Die Homomorphismen $\varphi a$ und $\hat{\psi} i^{-1} p$ von $\hat{H}$ in $L$ lassen sich auf die Bohrkompaktifizierung $r(H)^{-}$von $\hat{H}$ fortsetzen. Die Fortsetzungen stimmen aber überein, da sie auf der dichten Untergruppe $r(H)$ übereinstimmen, also sind $\varphi a$ und $\hat{\psi} i^{-1} p$ gleich $\left(s: \hat{H} \rightarrow r(H)^{-}\right.$ist injektiv!). Insbesondere ist Kern $\varphi a=\operatorname{Kern} \hat{\psi} i^{-1} p$, man erhält $H \cap \widehat{K}=\operatorname{Kern} \varphi a=\operatorname{Kern} \hat{\psi} i^{-1} p \supset \operatorname{Kern} p=\hat{K} \quad$ und schließlich $H=\hat{H}$, wenn man noch $\hat{H}=H \hat{K}$ berücksichtigt.

\section{§ 3. [MOORE]-Gruppen und die Klassen $\mathfrak{A}$ und $\mathfrak{D}$}

Aus (2.14) folgt nun leicht:

(3.1) Satz. Jede [TAK]-Gruppe liegt in $\mathfrak{A} \cap \mathfrak{D}$.

Beweis. Sei $G$ eine [TAK]-Gruppe. Die topologische Kommutatorgruppe $\bar{G}^{\prime}$ ist ein kompakter Normalteiler in $G$, und $G / \bar{G}^{\prime}$ liegt als lokalkompakte abelsche Gruppe in $\mathfrak{U} \cap \mathcal{D}$. Da $G$ ferner nach Voraussetzung eine [MAP]-Gruppe ist, liegt $G$ nach (2.14) in $\mathfrak{A} \cap \mathfrak{D}$.

(3.2) Bemerkung. Nach Theorem 3.1 von [6] und Korollar 1 $\mathrm{zu}$ Theorem 4.4 von [5] ist jede [Z]-Gruppe eine [TAK]-Gruppe und liegt mithin in $\mathfrak{A} \cap \mathfrak{D}$. Insbesondere gibt es daher zu jeder abgeschlossenen zentralen Untergruppe $H$ einer $[Z]$-Gruppe $G$ und zu jedem Charakter $\varphi$ von $H$ (den man als eindimensionale Darstellung von $H$ auffasse) eine endlichdimensionale irreduzible Darstellung $\varrho$ von $G$ in $V$ mit $\varrho(x)=\varphi(x) 1_{V}$ für alle $x \in H$. Dies ist die Aussage von Theorem 5.5 in [6], welche sich hier als Spezialfall eines allgemeineren Satzes erweist. 
(3.3) Bemerkung. Ferner folgt aus (3.1), daß Quotienten von [TAK]-Gruppen ebenfalls in [TAK] liegen; denn Gruppen in $\mathfrak{A}$ (oder $\mathfrak{D}$ ) sind [MAP]-Gruppen.

Als nächstes wollen wir einen einfachen Beweis des Robertsonschen Satzes über die Struktur von [MOORE]-Gruppen geben (vgl. [16] oder [9], p. 233), aus dem dann folgt, daß jede [MOORE]Gruppe in $\mathfrak{A} \cap \mathfrak{D}$ liegt.

(3.4) Satz. Eine lokalkompakte Gruppe G ist dann und nur dann eine [MOORE]-Gruppe, wenn $G$ eine abgeschlossene, in [TAK] gelegene, (normale) Untergruppe von endlichem Index enthält.

(3.5) Korollar. Jede [MOORE]-Gruppe liegt in $\mathfrak{A} \cap \mathfrak{D}$.

Das Korollar folgt unmittelbar aus dem Satz, aus (3.1) und aus (2.12).

Beweis von (3.4). Da es sich um den Beweis eines bekannten Satzes handelt, sei vorab eine Übersicht über die Sätze gegeben, die wir im Beweis verwenden wollen. Einen großen Teil dieser Sätze werden wir auch an anderer Stelle benutzen, der betreffende Satz wird dort mit $(X)$ aus (3.4) zitiert, $X=A-G$.

$(A)$ : Eine zusammenhängende [MAP]-Gruppe ist isomorph zu einem direkten Produkt aus einer kompakten Gruppe und einem $\mathbb{R}^{n}$ (vgl. etwa p. 195 in [7] oder p. 145 in [8]).

$(B)$ : Ist $G$ eine [MAP]-Gruppe und $G_{0}$ isomorph zu einem $\mathbb{R}^{n}$, so ist der Zentralisator von $G_{0}$ in $G$ von endlichem Index in $G$ (Theorem 4 in [18]).

$(C)$ : Ist $G$ eine [MOORE]-Gruppe, so hat $G$ kleine invariante Umgebungen der 1, und es gibt daher einen kompakten Normalteiler $K$ in $G$ so, daB $G / K$ eine Liegruppe ist (4.2 und 4.3 in [12]).

$(D)$ : Ist $\pi$ eine irreduzible Darstellung einer Gruppe $G$ und $\varrho$ eine endlichdimensionale Darstellung von $G$, so ist $\pi \otimes \varrho$ eine endliche Summe irreduzibler Teildarstellungen (Korollar zu dem Theorem in [14]).

$(E)$ : Ist $\pi$ eine irreduzible Darstellung einer Gruppe $G$ und $H$ eine Untergruppe von endlichem Index; so ist die Restriktion von $\pi$ auf $H$ eine endliche Summe irreduzibler Teildarstellungen (Proposition 2 in [14]).

$(F)$ : Ist $G$ eine diskrete Gruppe in [MOORE], so ist $G$ vom Typ I (Theorem 7 in [10]) und enthält daher einen abelschen Normalteiler von endlichem Index (Satz 4 in [17]). 
$(G)$ : Ist $G$ eine [MOORE]-Gruppe und $H$ eine offene Untergruppe von endlichem Index in $G$, so ist auch $H$ eine [MOORE]Gruppe (folgt sofort aus dem Reziprozitäts-Satz von Frobenius, vgl. auch 2.1 in [12]).

Sei nun $G$ eine [MOORE]-Gruppe. Nach $(C)$ gibt es einen kompakten Normalteiler $R$ in $G$ so, daß $G / R$ eine Liegruppe ist. $G / R$ ist eine [MOORE]-Gruppe, daher ist $(G / R)_{0}$ eine [MAP]Gruppe, und es gibt wegen $(A)$ einen kompakten zusammenhängenden Normalteiler $S$ in $G / R$ derart, daß $[(G / R) / S]_{0}$ isomorph zu einem $\mathbb{R}^{n}$ ist. Zusammen folgt: Fs gibt einen kompakten Normalteiler $K$ in $G$ derart, daß $L:=G / K$ eine Liegruppe und $L_{0}$ isomorph zu einem $\mathbb{R}^{n}$ ist. Nach $(F)$ und $(B)$ gibt es einen offenen Normalteiler $M$ von endlichem Index in $L$ derart, daß $M / L_{0}$ abelsch und $L_{0}$ zentral in $M$ ist. Ferner ist $M$ nach $(G)$ eine [MOORE]-Gruppe. Der erste Teil von (3.4) ist bewiesen, wenn wir gezeigt haben, daß $M$ abelsch ist. Wir nehmen an, daß die Kommutatorgruppe $M^{\prime}$ nicht trivial ist. $M^{\prime}$ ist in $M_{0}=L_{0} \cong \mathbb{R}^{n}$ enthalten und mithin eine unendliche Gruppe. Es gibt dann eine abgeschlossene Untergruppe $U$ von $M_{0}$ derart, daß $M_{0} / U$ isomorph zur Gruppe $T$ der komplexen Zahlen vom Betrage 1 ist und daß $M^{\prime} U / U$ dicht in $M_{0} / U$ ist (für $U$ wählt man etwa den Kern eines Charakters $\chi$ von $M_{0}$ mit $\left.\chi\left(M^{\prime}\right)^{-}=T\right)$. Sei dann $F:=M / U(U$ ist zentral in $M)$. Offenbar ist $\bar{F}^{\prime}$ zentral in $F^{\prime}$ und isomorph zu $T$. Ferner ist $F$ eine [MOORE]und daher insbesondere eine [MAP]-Gruppe. Nun gilt aber:

(3.6) Lemma. Sei o eine irreduzible Darstellung der lokalkompakten Gruppe $G$ in dem endlichdimensionalen Hitbertraum $V$. Ist $H$ eine Untergruppe von $\overline{G^{\prime}} \cap Z G$ und $n$ die Dimension von $V$, so ist $\varrho\left(x^{n}\right)=1_{V}$ für alle $x \in H$.

Beweis von (3.6). Da $H$ im Zentrum von $G$ liegt und $\varrho$ irreduzibel ist, gibt es einen Charakter $\chi$ von $H$ mit $\varrho(x)=\chi(x) 1_{V}$ für alle $x \in H$. Da $H$ in $\bar{G}^{\prime}$ liegt, ist $\varrho(H)$ in der speziellen unitären Gruppe von $V$ enthalten. Zusammen folgt die Behauptung.

Aus (3.6) folgt, daß die Restriktion einer irreduziblen endlichdimensionalen Darstellung $\varrho$ von $\bar{F}$ auf $\bar{F}^{\prime}$ trivial ist, d. h. daß $\bar{F}^{\prime}$ in Kern $\varrho$ liegt; im Widerspruch zur Tatsache, da $B F$ eine [MAP]Gruppe ist. Daher ist $M^{\prime}$ trivial, und eine Richtung von (3.4) ist bewiesen.

Für die Umkehrung genügt es wegen $(E)$ zu zeigen, daß jede [TAK]-Gruppe eine [MOORE]-Gruppe ist. Sei also $\pi$ eine irredu- 
zible Darstellung der [TAK]-Gruppe $G$ in dem Hilbertraum $\mathfrak{H}$. Da $\bar{G}^{\prime}$ kompakt ist, enthält die Restriktion $\pi \mid \bar{G}^{\prime}$ eine endlichdimensionale irreduzible Teildarstellung $\pi_{1}$. Nach (3.1) gibt es eine endlichdimensionale irreduzible Darstellung $\varrho$ von $G$ in $V$ derart, daß die duale Darstellung $\pi_{1}^{*}$ von $\pi_{1}$ in $\varrho / \vec{\sigma}^{\prime}$ enthalten ist. Da $\pi_{1} \otimes \pi_{1}^{*}$ die triviale Darstellung (von $\bar{G}^{\prime}$ ) enthält, ist der Raum

$$
\mathfrak{H}_{0}:=\left\{h \in \mathfrak{H} \otimes V \mid(\pi(g) \otimes \varrho(g))(h)=h \quad\left(\forall g \in \bar{G}^{\prime}\right)\right\}
$$

von Null verschieden. $\mathfrak{S}_{0}$ ist ein abgeschlossener, unter $(\pi \otimes \varrho)(G)$ invarianter Teilraum von $\mathfrak{H} \otimes V$. Wegen $(D)$ ist die Einschränkung $\tau$ von $\pi \otimes \varrho$ auf $\mathfrak{S}_{0}$ eine endliche Summe irreduzibler Teildarstellungen. Da aber $\tau$ über $G \rightarrow G / \bar{G}^{\prime}$ faktorisiert und irreduzible Darstellungen von $G / \bar{G}^{\prime}$ eindimensional sind, ist $\mathfrak{H}_{0}$ ein endlichdimensionaler Teilraum. Dem Projektor $P$ von $\mathfrak{H} \otimes V$ auf $\mathfrak{H}_{0}$ entspricht ein von Null verschiedenes Element $J P \in \mathrm{Hom}_{G}\left(\pi, \pi \otimes \varrho \otimes \varrho^{*}\right)(\mathrm{vgl}$. [14]) mit endlichdimensionalem Bild. Da $\pi$ irreduzibel ist, ist JP injektiv und mithin $\mathfrak{S}$ endlichdimensional, q. e. d.

Nach Theorem 2 aus [12] enthält jede Liegruppe in [MOORE] eine offene, in $[Z]$ gelegene Untergruppe von endlichem Index. Der nächste Satz gibt eine etwas genauere Beschreibung der Struktur dieser Gruppen. Ferner kann dieser Satz als Verallgemeinerung des Struktursatzes für kompakte zusammenhängende Liegruppen angesehen werden, der beim Beweis auch eine Schlüsselrolle einnimmt.

(3.7) Satz. Sei G eine Liegruppe in [MOORE]. Es gibt dann einen offenen Normalteiler $H$ von endlichem Index in $G$ mit $H=\bar{H}^{\prime} Z H$, und $\bar{H}^{\prime}$ ist eine kompakte halbeinfache zusammenhängende Liegruppe (insbesondere ist $\bar{H}^{\prime} \cap Z H$ endlich).

Beweis. Sei $N$ ein offener Normalteiler von endlichem Index in $G$ derart, daß $\bar{N}^{\prime}$ kompakt ist $((3.4)) \cdot \bar{N}^{\prime}$ hat als kompakte Liegruppe eine treue endlichdimensionale Darstellung $\sigma$. Da $G$ in $\mathscr{D}$ liegt, gibt es eine endlichdimensionale Darstellung $\tau$ von $G$ derart, daß $\sigma$ in der Restriktion von $\tau$ auf $\bar{N}^{\prime}$ enthalten ist, insbesondere ist $\left.\tau\right|_{\bar{N}}$, injektiv (vgl. auch Korollar $3 \mathrm{zu}$ Theorem 5.1 in [6]). Wir fassen nun $\tau$ auf als stetigen Homomorphismus von $G$ in die kompakte Liegruppe $\tau(G)^{-}=K$. Sei $L$ die Zusammenhangskomponente des Einselementes in $K$. Dann ist $H:=N \cap \tau^{-1}(L)$ ein offener Normalteiler von endlichem Index in $G$, und die Restriktion und Corestriktion $\varphi$ von $\tau$ auf $H$ bzw. $L$ ist ein stetiger dichter Homomorphismus von $H$ in $L$, der 
auf $\bar{H}^{\prime} \subset \bar{N}^{\prime}$ treu ist. Wegen der Kompaktheit von $\bar{H}^{\prime}$ ist $\varphi\left(\bar{H}^{\prime}\right)=$ $=\overline{\varphi\left(H^{\prime}\right)}$. Da $\varphi$ dicht ist, ist $\overline{\varphi\left(H^{\prime}\right)}$ normal in $L$ und $\overline{L / \varphi\left(H^{\prime}\right)}$ abelsch. Also ist $L^{\prime}$ in $\overline{\varphi\left(H^{\prime}\right)}$ enthalten. Da $L^{\prime}$ abgeschlossen ist, gilt auch die andere Inklusion und mithin $L^{\prime}=\overline{\varphi\left(H^{\prime}\right)}=\varphi\left(\bar{H}^{\prime}\right)$. Nach dem Struktursatz für kompakte zusammenhängende Liegruppen (vgl. [8], Chap. XIII, Theorem 1.3., p. 144) ist $L^{\prime}$ eine kompakte zusammenhängende halbeinfache Liegruppe (und mithin auch die zu $L^{\prime}$ isomorphe Gruppe $\bar{H}^{\prime}$ - womit der zweite Teil des Satzes bewiesen ist), und es gilt $L=L^{\prime} Z L$. Wir sind daher fertig, wenn wir zeigen können, daß $\varphi^{-1}(Z L)$ in $Z H$ liegt. Ist nun $x \in \varphi^{-1}(Z L), y$ ein beliebiges Element in $H$ und $[x, y]$ der Kommutator von $x$ und $y$, so gilt $\varphi([x, y])=[\varphi(x), \varphi(y)]=e($ da $\varphi(x) \in Z L)$ und mithin $[x, y]=e$ (da $\left.\varphi\right|_{\bar{H}^{\prime}}$ injektiv ist).

Nach (5.1) von [4] besitzt jede kompakt erzeugte Liegruppe in $[Z]$ eine treue endlichdimensionale Darstellung. Wir wollen hier einen einfachen Beweis für den (unbedeutend allgemeineren) Fall geben, daB die Gruppe eine [MOORE]-Gruppe ist.

(3.8) Satz. Sei $G$ eine kompakt erzeugte Liegruppe in [MOORE]. Dann hat $G$ eine treue endlichdimensionale Darstellung.

Beweis. $G$ enthält nach (3.4) einen offenen Normalteiler $H$ von endlichem Index, welcher eine [TAK]-Gruppe ist. Offensichtlich genügt es zu zeigen, daß $H$ eine treue endlichdimensionale Darstellung besitzt. Mit $G$ ist auch $H$ kompakt erzeugt; denn ist $G=\bigcup_{i=1}^{s} H g_{i}$ (mit $g_{1}=e$ ) und wird $G$ von $K$ erzeugt, so überlegt man sich leicht, daß $H$ von $\bigcup_{i, j=1}^{s} H \cap g_{i} K g_{j}^{-1}$ erzeugt wird. Ferner besitzt $H$ eine endlichdimensionale Darstellung $\sigma$, deren Restriktion auf $\bar{H}^{\prime}$ treu ist (vgl. Beweis zu (3.7)). Mit $H$ ist auch $H / \bar{H}^{\prime}$ eine kompakt erzeugte Liegruppe. Die Struktur der lokalkompakten abelschen, kompakt erzeugten (Lie-)Gruppen ist wohlbekannt (vgl. etwa Satz 51 in [15]). Insbesondere gibt es eine endlichdimensionale Darstellung $\tau$ von $H$ mit $\operatorname{Kern} \tau=\bar{H}^{\prime}$. Dann ist aber $\sigma \oplus \tau$ eine treue endlichdimensionale Darstellung von $H$.

Um zu zeigen, daß jede lokalkompakte auflösbare Gruppe in $\mathscr{D}$ eine [MOORE]-Gruppe ist, benötigen wir den folgenden Satz:

(3.9) Satz. Sei G eine lokalkompakte Gruppe, $N$ ein abgeschlossener abelscher Normalteiler in $G$. G operiert auf der Charaktergruppe $\hat{N}$ von $N$ durch $(g \cdot \chi)(x)=\chi\left(g^{-1} x g\right)$ für $g \in G, x \in N$ und $\chi \in \hat{N}$. Für 
jeden Charakter $\chi \in \hat{N}$ sei die Bahn $G_{\chi}$ endlich. Sei $\pi$ eine Faktordarstellung von $G$ in dem Hilbertraum $\mathfrak{H}$. Dann gibt es einen Charakter $\chi \in \hat{N}$ derart, daß $\mathfrak{H}$ die (orthogonale) Summe der abgeschlossenen Unterräume $\mathfrak{S}_{\eta}:=\{h \in \mathfrak{H} \mid \pi(x) h=\eta(x) h$ fürr alle $x \in N\}, \eta \in G \chi$, ist.

(3.10) Bemerlang. Sind $\mathfrak{S}$ und $G$ separabel, so ergibt sich (3.9) leicht aus der Mackeyschen Theorie (vgl. [1] oder [11]); denn $\hat{N}$ ist dann ein Standard-Borel-Raum und $\hat{N} / G$ ist abzählbar separiert, woraus folgt, daß der zu der Restriktion von $\pi$ auf $N$ gehörige Quasi-Orbit transitiv ist.

Beweis von (3.9). Im Beweis werden wir einige elementare Sätze aus der (Darstellungs-) Theorie der $C^{*}$-Algebren verwenden. Diese findet man etwa in [3]. Es sei $\lambda=\left.\pi\right|_{N} . Z u \lambda$ korrespondiert eine nicht ausgeartete ${ }^{*}$-Darstellung $\lambda^{\prime} \operatorname{der} C^{*}$-Algebra $C^{*}(N)$ von $N$ in $\mathfrak{H}$. Mit $B$ sei das Bild von $\lambda^{\prime}$ im Raum $B(\mathfrak{H})$ der beschränkten Operatoren auf $\mathfrak{S}$ bezeichnet. $B$ ist eine (kommutative) $C^{*}$-Algebra, $\lambda^{\prime}$ induziert einen surjektiven Morphismus $\varrho$ von $C^{*}(N)$ auf $B$. Mit $d x$ sei das Haarsche Maß auf $N$ bezeichnet. Es gelte $d\left(g x g^{-1}\right)=$ $=\Delta(g) d x$ für $g \in G$, d. h. für $f \in C_{k}(N)$, dem Raum der stetigen Funktionen auf $N$ mit kompaktem Träger, gilt

$$
\int_{N} f\left(g^{-1} x g\right) d x=\int_{N} f(x) \Delta(g) d x .
$$

$\Delta$ ist eine Abbildung von $G$ in die multiplikative Gruppe der positiven reellen Zahlen mit $\Delta\left(g_{1} g_{2}\right)=\Delta\left(g_{1}\right) \Delta\left(g_{2}\right)$ für $g_{1}, g_{2} \in G$. $G$ operiert auf $C_{k}(N)$ durch $(g \cdot f)(x)=\Delta(g)^{-1} f\left(g^{-1} x g\right)$. Man überlegt sich Jeicht, daß $G$ die 1-Norm erhält, daß $G$ linear operiert und mit der Faltung und der Involution verträglich ist. $G$ operiert mithin auch durch *-Automorphismen auf $C^{*}(N)$. Ferner gilt $\lambda^{\prime}(g \cdot f)=$ $=\pi(g) \lambda^{\prime}(f) \pi(g)^{*}$ für alle $g \in G$ und alle $f \in C^{*}(N)$. Man braucht diese Gleichung nur für $f \in C_{k}(N)$ nachzuweisen:

$$
\begin{gathered}
\lambda^{\prime}(g \cdot f)=\int_{N}(g \cdot f)(x) \lambda(x) d x= \\
=\int_{N} \Delta(g)^{-1} f\left(g^{-1} x g\right) \pi(x) d x=\int_{N} \Delta(g)^{-1} f(x) \pi\left(g x g^{-1}\right) d\left(g x g^{-1}\right)= \\
=\int_{N} f(x) \pi(g) \pi(x) \pi(g)^{*} d x=\pi(g) \int_{N} f(x) \pi(x) d x \pi(g)^{*}= \\
=\pi(g) \lambda^{\prime}(f) \pi(g)^{*} .
\end{gathered}
$$

Insbesondere gilt $\pi(g) B \pi(g)^{*}=B$ für alle $g \in G$; $G$ operiert damit durch *-Automorphismen auch auf $B$, und $\varrho$ ist $G$-äquivariant. 
$G$ operiert dann auch durch Homöomorphismen auf den Spektren $C^{*}(N)^{\wedge}$ bzw. $\hat{B}$ der kommutativen $C^{*}$-Algebren $C^{*}(N)$ bzw. $B$ (durch $(g \cdot \chi)(f)=\chi\left(g^{-1} \cdot f\right)$ für einen Charakter $\chi$ von $C^{*}(N)$, entsprechend für $B$ ). Man verifiziert leicht, daß die durch $\varrho$ induzierte injektive Abbildung $\hat{\varrho}$ von $\widehat{B}$ in $C^{*}(N)^{\wedge} G$-äquivariant ist. Ferner ist $C^{*}(N)^{\wedge}$ kanonisch homöomorph zu $\widehat{N}$, und der kanonische Homöomorphismus ist $G$-äquivariant (bezüglich der in (3.9) angegebenen Operation von $G$ auf $\hat{N}$ ). Insbesondere sind alle $G$ Bahnen in $\widehat{B}$ endlich. Wir verwenden nun das folgende Lemma, welches wir im Anschluß an den Beweis von (3.12) beweisen werden.

(3.11) Lemma. Sei $X$ ein nicht-leerer lokalkompakter HausdorffRaum. Eine (diskrete) Gruppe $G$ operiere durch Homöomorphismen auf $X$. Für jedes $x \in X$ sei die Bahn $G x$ endlich. Dann gibt es entweder ein $x_{0} \in X$ mit $G x_{0}=X$, oder es gibt eine nicht-konstante stetige Funktion $f$ mit kompaktem Träger von $X$ in das Einheitsintervall $[0,1]$ mit $f\left(g^{-1} x\right)=f(x)$ für alle $x \in X$ und alle $g \in G$.

Wir wenden (3.11) an auf $X=\hat{B}$. Nehmen wir zunächst an, daß der 2. Fall eintritt, d. h. daß es eine nicht-konstante, $G$-invariante stetige Funktion $f$ auf $\widehat{B}$ mit kompaktem Träger gibt. Die GelfandTransformation $\mathfrak{S}$ von $B$ auf die $C^{*}$-Algebra $C_{0}(\hat{B})$ der im Unendlichen verschwindenden, komplex-wertigen stetigen Funktionen auf $\hat{B}$ ist ein $G$-äquivarianter *-Isomorphismus (wobei $G$ auf $C_{0}(B)$ durch $(g \cdot f)(\chi)=f\left(g^{-1} \chi\right)$ wirkt). Es gibt folglich einen Operator $T \in B$ mit $\pi(g) T \pi(g)^{*}=T$ für alle $g \in G$, und $\mathfrak{I}(T)=f$ ist eine nicht-konstante Funktion in $C_{0}(\hat{B})$. $T$ liegt also in der Kommutante $\pi(G)^{\prime}$ von $\pi(G)$. Andererseits liegt $T$ in $B$, also in $\pi(N)^{\prime \prime}$ und erst recht in $\pi(G)$ ". Da $\pi$ eine Faktordarstellung ist, ist dann $T$ ein Vielfaches der Identität auf $\mathfrak{S}$, im Widerspruch zu der Tatsache, daß $\mathfrak{I}(T)$ eine nicht-konstante Funktion ist.

In unserer speziellen Situation tritt also der 1. Fall von (3.11) ein. Der Rest des Beweises (bis auf den noch ausstehenden Beweis von (3.11)) ergibt sich offensichtlich aus:

(3.12) Lemma. Sei $A$ eine kommutative $C^{*}$-Algebra, $\tau$ eine nichtausgeartele ${ }^{*}$-Darstellung von $A$ in dem Hilbertraum $\mathfrak{H}$. $\tau$ induziert einen surjektiven *-Morphismus $\varrho$ von $A$ auf die $C^{*}$-Algebra $B:=\tau(A)$. @ induziert eine injektive Abbildung @ von dem Spektrum $\widehat{B}$ von $B$ in das Spektrum $\hat{A}$ von $A$. Es sei $\varrho(\hat{B})=\left\{\chi_{1}, \ldots, \chi_{n}\right\}$ mit $\chi_{i} \neq \chi_{j}$ für $i \neq j$. Dann ist $\mathfrak{S}$ die orthogonale Summe der $\mathfrak{S}_{i}:=$ $=\left\{x \in \mathfrak{H} \mid \tau(a)(x)=\chi_{i}(a) x(\forall a \in A)\right\}$. 
Beweis von (3.12). Es sei $\widehat{B}=\left\{\eta_{1}, \ldots, \eta_{n}\right\}$ mit $\hat{\varrho}\left(\eta_{i}\right)=\eta_{i} \varrho=\chi_{i}$ für $1 \leqslant i \leqslant n$. Aus der Gelfand-Darstellung von $B$ folgt, daß es Projektoren $p_{1}, \ldots, p_{n}$ in $B$ gibt mit

(i) $p_{i} p_{j}=\delta_{i j} p_{i}$ für $1 \leqslant i, j \leqslant n$

(ii) $b=\sum_{i=1}^{n} \eta_{i}(b) p_{i} \quad(\forall b \in B)$

(iii) $\sum_{i=1}^{n} p_{i}=1_{\mathfrak{g}}(\mathrm{da} \tau$ nicht-ausgeartet ist).

Man verifiziert, $\operatorname{da} \mathfrak{H}_{i}=p_{i} \mathfrak{H}$ ist, womit (3.12) bewiesen ist.

Beweis von (3.11). Für eine endliche Menge $E$ sei $|E|$ die Anzahl der Elemente in $E$. Für jede natürliche Zahl $n$ sei dann $X_{n}:=\{x \in X|| G x \mid \leqslant n\}$. Nun gilt:

(a) Ist $x \in X,|G x|=m$, etwa $G x=\left\{g_{1} x, \ldots, g_{m} x\right\}$, so gibt es eine offene Umgebung $U$ von $x$ in $X$ derart, daß die Elemente $g_{1} u, \ldots, g_{m} u$ für alle $u \in U$ paarweise verschieden sind.

Man wähle nämlich etwa $U=\bigcup_{\substack{i, j=1 \\ i \neq j}}^{m}\left\{y \in X \mid g_{i} y \neq g_{j} y\right\}$. Aus (a) folgt, da $\beta$ das Komplement von $X_{n}$ offen ist; die Mengen $X_{n}$ sind mithin abgeschlossen in $X$. Ferner ist $X$ nach Voraussetzung gleich der Vereinigung der $X_{n}$. Da $X$ lokalkompakt ist, gibt es eine natürliche Zahl $m$ derart, daß das Innere $\dot{X}_{m}$ von $X_{m}$ nicht leer ist. Es sei $s:=\max |G x|$. Wähle nun ein für den Rest des Beweises festes $x_{0} \in \dot{\mathrm{X}}_{m}^{\substack{x \in \dot{\mathrm{X}}_{m} \\ \text { mit }}}\left|G x_{0}\right|=s$, etwa $G x_{0}=\left\{g_{1} x_{0}, \ldots, g_{s} x_{0}\right\}$. Mit (a) ergibt sich nun:

(b) Es gibt eine offene Umgebung $U$ von $x_{0}$ in $X$ mit $|G u|=s$ und $G u=\left\{g_{1} u, \ldots, g_{s} u\right\}$ für alle $u \in U$.

Es sei $v: X \rightarrow X / G$ die natürliche Abbildung auf den Bahnenraum, $X / G$ trage die Quotiententopologie. Ferner sei eine offene Umgebung $U$ von $x_{0}$ gemäß (b) gewählt.

1. Fall: $U \subset G x_{0}$ (d. h. $v(U)$ besteht aus einem Punkt). Dann ist $G x_{0}$ offen und abgeschlossen in $X$. Definiere $f: X \rightarrow[0,1]$ durch $\left.f\right|_{G x_{0}}=1$ und $\left.f\right|_{X \backslash G x_{0}}=0$. $f$ ist eine stetige, $G$-invariante Funktion mit kompaktem Träger. Entweder ist $X=G x_{0}$ oder $f$ ist nicht konstant, und wir sind fertig.

2. Fall: $v(U)$ besteht aus mindestens 2 Punkten. 
Wir zeigen zunächst: $v(U)$ ist offen in $X / G$. Der Unterraum $v(U)$ von $X / G$ ist ein lokalkompakter Hausdorff-Raum. Ist $u \in U$ mit $G u \neq G x_{0}$, so gibt es $K \subset v(U)$ mit

(i) $K$ ist kompakt.

(ii) $K$ ist abgeschlossen in $X / G$.

(iii) $v\left(x_{0}\right)$ liegt im Innern $K$ von $K$.

(iv) $v(u) \notin K$.

(v) $v^{-1}(K)$ ist kompakt in $X$.

Beweis. $v(U)$ ist offen, da $v^{-1}(\nu(U))=G U$ offen in $X$. Seien nun $x, y \in U$ mit $G x \neq G y$. Es gibt dann offene Mengen $V^{\prime}$ und $W^{\prime}$ in $X$ mit $x \in V^{\prime} \subset U, y \in W^{\prime} \subset U$ und $g_{i} V^{\prime} \cap g_{j} W^{\prime}=\emptyset$ für $i, j=1, \ldots, s$. Wähle sodann relativ kompakte, offene Mengen $V$ und $W$ mit $x \in V \subset \bar{V} \subset V^{\prime}$ und $y \in W \subset \bar{W} \subset W^{\prime}$. Dann ist $v(V) \cap \nu(W)=\emptyset$. Damit ist gezeigt, daß $v(U)$ ein Hausdorff-Raum ist. Ferner ist $v(\bar{V})$ eine kompakte Umgebung von $v(x)$ in $v(U) ; v(U)$ ist daher lokalkompakt. Ist $u \in U$ mit $G u \neq G x_{0}$ wie in der Behauptung vorgelegt, so führe man die obige Wahl von Umgebungen für $x=x_{0}$ und $y=u$ durch. Setze dann $K:=v(\bar{V})$. Offensichtlich sind (i), (iii) und (iv) erfüllt. Ferner ist $\nu^{-1}(K)=\nu^{-1}(\nu(\bar{V}))=G \bar{V}=\bigcup_{i=1}^{s} g_{i} \bar{V}$ (nach
(b)), also ist $v^{-1}(K)$ kompakt; (ii) folgt aus (v).

Wähle nun ein $u \in U$ mit $G u \neq G x_{0}$ und ein zugehöriges $K$ mit (i) - (v). Auf dem lokalkompakten $\operatorname{Raum} v(U)$ gibt es eine stetige Funktion $\varphi: v(U) \rightarrow[0,1]$ mit $\varphi\left(\nu\left(x_{0}\right)\right)=1$ und $\varphi(\nu(U) \backslash K)=\{0\}$. Setze $\varphi$ fort zu $\tilde{\varphi}: X / G \rightarrow[0,1]$ dureh $\tilde{\varphi} \mid \nu(U)=\varphi$ und $\left.\tilde{\varphi}\right|_{X / G \backslash \nu(U)}=0$. Da $v(U)$ offen und $K$ abgeschlossen in $X / G$ ist, ist $\tilde{\varphi}$ stetig. $f:=\tilde{\varphi} v$ ist eine stetige, nicht-konstante Funktion auf $X$, deren Träger in der kompakten Menge $v^{-1}(K)$ liegt. Damit ist (3.11) bewiesen.

(3.13) Satz. Eine lokalkompakte auflösbare Gruppe G liegt genau dann in $\mathscr{D}$, wenn $G$ eine [MOORE]-Gruppe ist.

Beweis. Nach (3.5) liegt jede [MOORE]-Gruppe in $\mathfrak{D}$. Die Umkehrung wird durch Induktion über die Stufe von $G$ bewiesen. Ist $G$ abelsch, so ist jede irreduzible Darstellung von $G$ eindimensional und $G$ mithin eine [MOORE]-Gruppe. Ist $G$ auflösbar von der Stufe $n>1$, so enthält $G$ einen abgeschlossenen abelschen Normalteiler $N$ derart, daß $G / N$ von der Stufe $n-1$ ist. Nach (2.11) liegt auch $G / N$ in $\mathfrak{D}$ und ist mithin nach Induktionsvoraussetzung eine [MOORE]-Gruppe. Wir wollen (3.9) auf das Paar $(N, G)$ anwenden. Zunächst ist nachzuprüfen, daß für jeden Cha- 
rakter $\chi \in \hat{N}$ die Bahn $G \chi$ endlich ist. Ist $\varrho$ eine endlichdimensionale Darstellung von $G$ in $V$, so sei $V_{\eta}:=\{v \in V \mid \varrho(x) v=\eta(x) v(V x \in N)\}$ für $\eta \in \hat{N} . V_{\eta}$ ist nur für endlich viele $\eta \in \widehat{N}$ von Null verschieden. Da $G$ in $\mathscr{D}$ liegt, gibt es zu einem vorgelegten $\chi \in \hat{N}$ eine endlichdimensionale (irreduzible) Darstellung $\varrho$ von $G$ in $V$ mit $V_{\chi} \neq 0$. Ist dann $g \in G$, so rechnet man leicht nach, daß $V_{g x}=\varrho(g) V_{x} \neq 0$ ist. Daher ist $G \chi$ endlich. Sei nun $\pi$ eine irreduzible Darstellung von $G$ in $\mathfrak{H}$. Wir haben zu zeigen, daß $\mathfrak{H}$ endlichdimensional ist. Nach (3.9) gibt es einen Charakter $\eta \in \widehat{N}$ derart, daß $\mathfrak{H}_{\eta}:=$ $=\{h \in \mathfrak{H} \mid \pi(x) h=\eta(x) h(\forall x \in N)\}$ von Null verschieden ist. Da $G$ in D liegt, gibt es eine endlichdimensionale (irreduzible) Darstellung $\varrho$ von $G$ in $V$ mit $V_{\bar{n}} \neq 0$. Nun betrachte man die Darstellung $\pi \otimes \varrho$ in $\mathfrak{S} \otimes V$. Auf $\mathfrak{H}_{\eta} \otimes V_{\bar{\eta}} \neq 0$ wirkt $N$ trivial, also ist $\mathfrak{S}_{0}:=$ $=\{h \in \mathfrak{H} \otimes V \mid(\pi \otimes \varrho)(x) h=h(\forall x \in N)\}$ von Null versehieden. $\mathfrak{H}_{0}$ ist ein abgeschlossener, unter $(\pi \otimes \varrho)(G)$ invarianter Unterraum. Wegen $(D)$ aus (3.4) ist die Restriktion $\tau$ von $\pi \otimes \varrho$ auf $\mathfrak{H}_{0}$ eine endliche Summe irreduzibler Teildarstellungen von $G$ bzw. von $G / N$. Da G/N eine [MOORE]-Gruppe ist, ist $\mathfrak{H}_{0}$ endlichdimensional. Wie im Beweis von (3.4) schließt man nun, daß auch $\mathfrak{H}$ endlichdimensional ist, und (3.13) ist bewiesen.

\section{Literatur}

[1] Austander, L., and C.C. Moone: Unitary Representations of Solvable Lie Groups. Memoirs Amer. Math. Soc. No. 62. Providence, R. I. 1966.

[2] BuRrow, M.: Representation Theory of Finite Groups. New York: Academic Press. 1965.

[3] Dixmrer, J.: Les $C^{*}$-algèbres et leurs représentations. Paris: Gauthier-Villars. 1969.

[4] Grosser, S., and M. Moskowitz: Compactness conditions in topological groups. J. Reine und Angew. Math. 246, 1-40 (1971).

[5] Grossme, S., and M. Moszowrtz: On central topological groups. Trans. Amer. Math. Soc. 127, 317-340 (1967).

[6] Grosser, S., and M. Mosxowitz: Representation theory of central topological groups. Trans Amer. Math. Soc, 129, 361-390 (1967).

[7] Heyer, H.: Dualität lokalkompakter Gruppen. Berlin-Heidelberg-New York: Springer. 1970.

[8] Hochschimd, G.: The Structure of Lie Groups. San Francisco: Holden-Day. 1965.

[9] KANIUTH, E.: Die Struktur der regulären Darstellung lokalkompakter Gruppen mit invarianter Umgebungsbasis der Eins. Math. Ann. 194, $225-248$ (1971). 
[10] Kaplansky, I.: Group algebras in the large. Tôhoku Math. J. (2) $3,249-256$ (1951).

[11] Mackey, G. W.: Unitary representations of group extensions I. Acta Math. 99, 265-311 (1958).

[12] Moons, C. C.: Groups with finite dimensional irreducible representations. Trans. Amer. Math. Soc. 166, 401-410 (1972).

[13] Pogun'TKe, D.: Einige Eigensehaften des Darstellungsringes kompakter Gruppen. Math. Z. 130, 107-117 (1973).

[14] Poguntke, D.: Decomposition of tensor products of irreducible unitary representations. Proc. Amor. Math. Soc. 52, 427-432 (1975). ner. 1958.

[15] Pontriagin, L. S.: Topologische Gruppen, Teil 2. Leipzig: Teub-

[16] Robertson, L. C.: A note on the structure of Moore groups. Bull. Amer. Math. Soe. 75, 594-599 (1969).

[17] Tнома, E.: Eine Charakterisierung diskreter Gruppen vom Typ I. Invent. Math. 6, 190-196 (1968).

[18] WrLcox, T.: On the structure of maximally almost periodic groups. Bull. Amer. Math. Soc. 73, 732-734 (1967).

Dr. D. PogunteKe

Fakultät für Mathematik

Universität Bielefeld

Kurt-Schumacher-Straße 6

D-4800 Bielefeld, Postfach 8640

Bundesrepublik Deutschland 